\title{
Acessibilidade de indivíduos infectados pelo HIV aos serviços de saúde: uma revisão de literatura
}

\author{
Accessibility to health services by HIV-infected \\ patients: a literature review
}

Francisco de Assis Acurcio 1

Mark Drew Crosland Guimarães 2

\footnotetext{
1 Departamento de Farmácia Social, Faculdade de Farmácia, Universi dade Federal de Minas Gerais. Av. Olegário Maciel 2360, 7o andar, Bel o Horizonte, MG, 30180-112, Brasil.

2 Departamento de Medicina Preventiva e Social, Faculdade de Medicina, Universidade Federal de Minas Gerais.

Av. Alfredo Balena 190, 9o andar, Belo Horizonte, MG 30130-100, Brasil.
}

Abstract The aim of this paper is to providea review of studies on accessibility to health care. It has been shown that patients infected by the human immunodeficiency virus (HIV) may present better survival esti mates depending upon their access to adequate health care, such as the availability of prophylactic therapy (e.g. zidovudine, pentamidine). The present revi ew has emphasized access to health care in light of data from the current HIV epi demic. Theorical aspects pertaining to accessibility are presented, followed by a bri ef literature review on four main topics: 1. epidemiological aspects of HIV; 2. evaluation of health service quality; 3. accessi bility to health care; and 4. accessibility studies on HIV-infected patients. Final considerations indicate the need to understand the various consequences of barriers to health care, taking into account the social and human costs of HIV infection, the needs and demands of HIV-infected patients, and the consequences of del ays in care for these individuals.

Key words HIV; AIDS; Health Services; Quality; Public Health

Resumo Este trabalho tem por objetivo apresentar uma revisão de estudos que enfocam o tema da acessi bili dade a ações e serviços de saúde. Tem-se demonstrado que indi vídu os infectados pelo vírus da imunodeficiência humana (HIV) podem apresentar uma mel hor sobrevi da dependendo do tipo de acesso que ten ham às ações e serviços de saúde, como, por exemplo, acesso a medicamentos profiláticos (por exemplo, zidovudina, pentamidina). Desta forma, o presente trabalho enfatiza e utiliza dados da epidemia do HIV como forma de abordar o tema proposto. O artigo inicia-se com uma exposição do marco teórico sobre acessibilidade, seguida de uma re visão bi bli ográfica estruturada em quatro tópicos, a saber: 1) aspectos epidemiológi cos da AIDS; 2) aval i ação de qual idade dos serviços de saúde; 3) acessi bi li dade às ações e servi ços de saú dee 4) estudos de acessibilidade em pacientes infectados pel o HIV. Consi derações finais sobre o tema enfocado a pontam para a necessi dade de se compreender as i mplicações das diversas barrei ras de acesso aos serviços, levando-se em conta os custos sociais e humanos da infecção pel o HIV, as necessi dades e demandas dos indivíduos infectados e as conseqüênci as que o não-atendimento em tempo hábil podeter para estes indivíduos.

Palavras-chave HIV; AIDS; Serviços de Saúde; Qualidade; Saúde Pública 


\section{Marco teórico}

Em uma primeira aproximação, os estudos de acessibilidade a ações e serviços de saúde se inserem no campo da Investigação de Serviços de Saúde, na área temática da Avaliação da Qualidade de Serviços de Saúde. Uma Investigação de Serviços de Saúde pode ser definida como "aquele estudo que tem por objeto o Sistema de Saúde ou al gum de seus componentes ou interrelações, com o propósito de fornecer elementos que permitam reorientá-los ou reorganizálos de maneira que as premissas de eqüidade, eficiência e eficácia sejam uma realidade no menor tempo possível" (Chorny et al.,1990).

Na opinião de Donabedian (1966), existe uma grande dificuldade de definir a qualidade da atenção. Os critérios de qualidade seriam juízos de valor que podem ser aplicados a distintos aspectos, propriedades, componentes ou alcances de um processo denominado atenção médica.

A acessibilidade, ou o acesso, a ações e serviços de saúde tem sido considerada como um dos componentes principais da qualidade da atenção (Somers,1971; Vuori,1988; Donabedian,1990b). Pode ser conceituada como a capacidade do paciente obter, quando necessitar, cuidado de saúde, de maneira fácil e conveniente. Entretanto, é pertinente incorporar o alerta de Aday \& Andersen (1974): “As distintas características do sistema e da população podem determinar a possibilidade de ingressar ao mesmo ou não, mas a prova do acesso em si não consiste na disponibilidade dos serviços e recursos, mas sim no fato de que os serviços sejam utilizados por quem os necessite."

Segundo o marco teórico para o estudo do acesso propugnado por Aday \& Andersen (1974), constata-se que, em termos gerais, as políticas de saúde têm estado orientadas a meIhorar o acesso aos serviços. Planificadores de serviços de saúde e dirigentes buscam avaliar o efeito que a política de saúde tem sobre o acesso à atenção e a possibilidade de modificá-lo.

O termo "sistema de prestação" é utilizado para referir-se à forma de organizar a prestação para a potencial atenção aos consumidores e se caracteriza por dois elementos principais: recursos e organização. Os recursos compreendem a mão de obra e o capital dedicados à atenção da saúde. A organização compreende o que o sistema faz com seus recursos e se refere à maneira como os agentes de saúde e os serviços existentes se coordenam e controlam no processo de oferecer serviços médicos.

As características da população em situação de risco são os componentes de predispo- sição, capacidade e necessidade. A predisposição compreende as variáveis que descrevem a propensão dos indivíduos a utilizarem os serviços. A capacidade se refere aos meios com que os indivíduos contam para utilizar os serviços. A necessidade se refere ao nível de enfermidade, que é a causa mais imediata da utilização dos serviços de saúde.

De acordo com Aday \& Andersen (1974), pode-se empregar o nível e modelo de utilização real do sistema como uma medida para comprovação do valor preditivo dos indicadores de acesso baseados no sistema e nos indivíduos. Pode-se caracterizar a utilização de serviços de saúde em termos de tipo, lugar, motivo e intervalo de tempo compreendido.

Finalmente, o grau de satisfação das necessidades do consumidor pode ser medido de acordo com a atitude para com o sistema de atenção médica, adotada pelos indivíduos que tenham tido contato com o mesmo.

As relações entre os diversos componentes do conceito de acesso, de acordo com a hipótese de Aday \& Andersen (1974), podem ser assim explicitadas:

- As políticas de saúde tendem a modificar diretamente as características do sistema de prestação e os programas tendem a modificar as características da população em situação de risco (diretamente ou por meio dos sistemas de prestação). As propriedades mutáveis da população em situação de risco podem, também, ser modificadas pela política de saúde.

- O sistema de prestação pode afetar os modelos de utilização e a satisfação que os consumidores experimentam com o sistema. Além disto, o referido sistema pode modificar, através de programas de educação para a saúde, as características da população e, assim, afetar indiretamente sua utilização dos serviços e a satisfação do consumidor com a atenção.

- As características da população podem afetar diretamente a utilização e a satisfação por parte dos consumidores, independentemente das propriedades do sistema.

- A utilização e satisfação por parte dos consumidores sugere uma seqüência na qual a utilização dos serviços pode afetar a satisfação de um paciente com o sistema, e, por sua vez, a satisfação ou descontentamento que este experimenta afeta sua utilização posterior dos serviços.

De acordo com Davis (1991), “o mais recente desenvolvimento na literatura de Investigação em Serviços de Saúde sobre acesso a cuidados de saúde é a tendência em direção a estudos mais desagregados, que olham para serviços específicos e a ligação entre acesso a serviços de saúde e resultados de saúde". 
O método, baseado na avaliação de resultado ou o estudo dos resultados finais, procura descobrir um aspecto mensurável do estado de saúde de um indivíduo ou de um grupo de indivíduos, além de determinar a variação que ocorreu no estado de saúde como resultado de condições que afetam o conteúdo, o processo e a estrutura da atenção à saúde. Assim, um critério fundamental para julgar a qualidade da atenção firma-se em uma alteração do estado de saúde de quem recebe a atenção (De Geyndt, 1970).

Nessa perspectiva, têm sido desenvolvidos estudos, enfocando indivíduos infectados pelo Vírus da I munodeficiência Humana (HIV), que se propõem a analisar os efeitos que barreiras ao acesso a serviços e ações de saúde podem ter sobre os resultados na saúde destes indivíduos. Uma vez que os indivíduos podem ser identificados como HIV positivos em um longo intervalo entre a infecção inicial e a doença sintomática, estudos envolvendo a progressão da doença e experiências de utilização de serviços são essenciais. Além disso, proporcionam uma interessante fusão de conceitos epidemiológicos básicos e metodologias de investigação de serviços de saúde.

\section{Revisão bibliográfica}

\section{Aspectos epidemiológicos da AIDS}

Atualmente a Síndrome da Imunodeficiência Adquirida (AIDS) se caracteriza como uma pandemia, em que a via sexual - principalmente heterossexual sem proteção - éa forma mais importante de transmissão do HIV, atingindo especialmente os países em desenvolvimento, que têm suportado os mai ores ônus. Esse quadro altera profundamente o panorama inicial da doença, quando a forma predominante de transmissão do vírus era por relações homossexuais sem proteção e pelo uso de drogas endovenosas. A epidemia aparece como um dos principais problemas de saúde pública para as próximas décadas. Já foram notificados mais de quinhentos mil casos de AIDS no mundo, desde 1981, quando foram descritos, nos Estados Unidos, os primeiros casos da doença. A Organização Mundial de Saúde estima que existem hoje no mundo mais de treze milhões de pessoas infectadas pelo HIV (Hayes, 1992). Projeções quanto ao curso da epidemia indicam que a incidência anual de novos casos de AIDS pode estabilizar-se em países da Europa e América do Norte. Ao contrário, o número anual de casos novos de AIDS continuará aumentando rapidamente em países da América
Latina e África. De acordo com o Relatório sobre o Desenvolvimento Mundial (Banco Mundial,1993), até o ano 2000, nos países em desenvolvimento, a mortalidade devido à AIDS pode subir, com facilidade, a mais de 1,8 milhão de mortes por ano, o que anularia décadas de esforço na conquista de reduções de mortalidade nesses países.

A epidemia de AIDS apresenta uma acentuada variação geográfica e racial, e os padrões epidemiológicos têm mudado rapidamente em muitos países.

O Brasil situa-se entre os quatro países com maior número de casos no mundo, ainda que, em termos de incidência acumulada, encontrava-se, em 1992, em vigésimo lugar [19 por 100.000 habitantes] (Guimarães \& Castilho 1993). Estimou-se que o número de infectados no país pelo HIV, em janeiro de 1992, na população de 15 a 45 anos, estivesse entre 280.000 a 420.000 indivíduos. De acordo com dados mais recentes divulgados pelo Ministério da Saúde (MS, 1995), a evolução dos casos notificados apresenta as seguintes características: 1) um grande aumento do número de casos, com 62.314 casos notificados até fevereiro de 1995; 2) a taxa de incidência acumulada de casos de AIDS aumentou de 0,005 por 100.000 habitantes, em 1982, para 45,9, em fevereiro de 1995; 3) a razão homem:mulher decresceu de 126:1, em 1984, para 4:1, em 1994/ 1995; 4) a região Sudeste é responsável por 77,3\% dos casos notificados até fevereiro de 1995, mas o número de casos cresce em todas as regiões; 5) para o sexo masculino, 56,1\% dos casos notificados até fevereiro de 1995 correspondem à transmissão sexual, 25,6\% à transmissão sangüínea e em $18,3 \%$ dos casos às categorias não-definidas; 6) para o sexo feminino, 39,0\% dos casos notificados até fevereiro de 1995 correspondem à transmissão sexual, 35,0\% à transmissão sangüínea e em $26,0 \%$ dos casos as categorias não são definidas; 7) as formas de transmissão foram se alterando no decorrer dos últimos quinze anos. Ao término do período de 1980 a 1987, as práticas homossexuais e bissexuais predominavam como vias de transmissão. Em 1994 as práticas homossexuais, o uso de drogas injetáveis e as práticas heterossexuais aparecem como as principais vias de transmissão; 8) a faixa etária mais atingida corresponde à população entre 20 e 44 anos, com $81,3 \%$ dos casos notificados até fevereiro de 1995, sendo que 59,4\% encontram-se entre 25 e 39 anos; 9) do total de casos notificados entre 1980 e 1994, tem-se conhecimento de 23.341 óbitos, o que representa um coeficiente de letalidade de $37.5 \%$; 10. entre os casos brasileiros de AIDS, 
candidíase, pneumonia por Pneumocystis carinii e tuberculose são as infecções oportunistas mais freqüentes: $40,1 \% ; 28,2 \%$ e $16,5 \%$, respectivamente.

A mediana de sobrevida após o diagnóstico é igual a 5,1 meses, enquanto nos países centrais a mediana de sobrevida é superior a 12 meses. Os pacientes mais jovens e aqueles usuários de drogas injetáveis apresentam uma sobrevida maior: 7,3 e 8,9 meses, respectivamente (Castilho et al., 1992). A sobrevida foi maior em pacientes que tiveram Sarcoma de Kaposi, candidíase, pneumonia por P. carinii e tuberculose, quando comparados com aqueles que tiveram toxoplasmose, como primeira doença indicativa de AIDS (Castilho et al., 1992). No período 1983-1986, para a cidade de São Paulo, estimou-se em 12,7\% de Anos Potenciais de Vida Perdidos (APVP) devido à AIDS, entre homens de 25 a 44 anos de idade (CastiIho et al., 1992). As projeções de número de casos de AIDS entre adultos de 15 a 45 anos para o ano de 1996 indicam limites inferior e superior de, respectivamente, 125.000 e 186.000 casos (Guimarães \& Castilho,1993).

Pesquisa realizada pela Datafolha e divulgada pela imprensa (Biancarelli, 1993), com 5.078 entrevistados em dez capitais do país, mostra a incorporação da AIDS ao cotidiano e ao imaginário da maioria da população: 38\% dos entrevistados declararam conhecer pessoalmente alguém que morreu de AIDS, 13\% admitem ter chances de estarem infectados pelo HIV e 39\% declararam mudanças de comportamento. Para a grande maioria, a AIDS amedronta e preocupa: 76\% têm medo de contrair a doença e $90 \%$ receiam que os filhos a contraiam. Os jovens de 16 a 25 anos são os que mais confessam ter medo (83\%). Quanto às expectativas de providências que o governo deveria tomar contra a doença, 58\% solicitam maior orientação pelos meios de comunicação; 16\% pedem melhor sistema de saúde e 9\% indicam investimentos em pesquisas e remédios. É digno de nota que 58\% acham que o governo não está fazendo quase nada para controlar a epidemia.

A avaliação de qualidade dos serviços de saúde

Donabedian vem trabalhando há vários anos com avaliação da atenção em saúde, particularmente em seus aspectos qualitativos. Utiliza três categorias de análise denominadas de estrutura, processo e resultado. Afirma que "no coração da investigação da qualidade está o esclarecimento da relação entre processo e resul- tado e entre a estrutura e o processo" (Donabedian, 1986).

O termo estrutura refere-se aos atributos materiais e organizacionais relativamente estáveis nos locais onde se proporciona a atenção (Donabedian, 1990a). Na abordagem estrutural, descrevem-se os recursos em termos de força de trabalho em saúde, instalações e equipamentos, e se comparam com critérios e padrões estabelecidos (Vuori, 1988).

O termo processo refere-se à atenção que médicos e outros provedores dispensam aos pacientes, assim como a habilidade com que efetuam essa atenção. Também se inclui aqui o que os pacientes fazem por si mesmos (Donabedian, 1990a).

Resultados expressam as conseqüências da atenção (ou da falta dela) na saúde de quem a recebe (ou deixa de receber) (Donabedian, 1986). O termo resultado refere-se ao que se obtém para o paciente e supõe uma alteração no estado de saúde que possa ser atribuída à atenção sob avaliação. Os resultados também incluem outras conseqüências da atenção, como por exemplo, conhecimento sobre a enfermidade, mudanças na conduta e a satisfação do paciente (Donabedian, 1990a).

Em outro artigo mais recente, Donabedian (1990b) considera que a qualidade do cuidado é um conceito que tem muitos componentes, os quais podem ser agrupados sob sete eixos: a) eficácia, b) efetividade, c) eficiência, d) otimização, e) aceitabilidade, f) legitimidade, h) eqüidade.

A eficácia é a capacidade da ciência e da arte do cuidado à saúde produzirem melhorias na saúde e bem-estar. A efetivi dade é a melhoria na saúde que se consegue, ou pode-se esperar conseguir, sob as circunstâncias rotineiras da prática diária. A eficiência é uma medida do custo para o qual qualquer melhoria de saúde é conseguida, enquanto que a otimização significa valorizar os efeitos do cuidado relacionados ao seu custo.

A aceitabilidade é entendida como uma adaptação do cuidado aos desejos, expectativas e val ores dos pacientes e de suas famílias. Em grande parte, a aceitabilidade depende das valorizações subjetivas do paciente referentes a efetividade, eficiência e otimização - mas não intei ramente. Alguns outros elementos entram no quadro: a acessibilidade do cuidado, os atributos da relação profissional-paciente e as características do local de prestação do cuidado. A acessibilidade é definida como a capacidade do paciente obter, quando necessitar, cuidado de saúde, de maneira fácil e conveniente. É um importante determinante na qualidade. A legi- 
timidade pode ser entendida como a aceitabilidade do cuidado para a comunidade ou a sociedade em geral.

Eqüidade é o princípio através do qual se determina o que é justo ou satisfatório na distribuição do cuidado e seus benefícios entre os membros da população. Como um dos pilares da qualidade, inclui: o que indivíduos consideram satisfatório; o que a sociedade considera satisfatório; a distribuição do acesso ao cuidado e a distribuição da qualidade do cuidado subseqüente e de suas conseqüências.

Na visão de Donabedian (1990b), a qualidade do cuidado é julgada pela sua conformidade com um grupo de expectativas ou padrões que derivam de três fontes: a) a ciência do cuidado à saúde que determina a eficácia; b) os valores e expectativas individuais que determinam a aceitabilidade e c) valores e expectativas sociais que determinam a legitimidade. Por conseqüência, a qualidade não pode ser inteiramente julgada em termos técnicos por profissionais de saúde isoladamente; as preferências de pacientes e da sociedade como um todo também devem ser levados em conta.

Vuori (1988) também aponta contribuições relevantes com relação ao tema ao enfatizar que, quando falamos sobre qualidade, é necessário especificar os aspectos da qualidade, qualidade definida por quem, e qualidade para quem. Assim, grupos portadores de diferentes interesses - planejadores de saúde, administradores, prestadores de serviço e os consumidores - podem expressar diferentes concepções do que constitui a melhor qualidade ou enfatizar diferentes aspectos da qualidade.

De modo semel hante à abordagem desenvolvida por Donabedian (1990b), Vouri destaca como componentes desejáveis do cuidado: a efetividade, a eficácia, a eficiência, a eqüidade, a acessibilidade, a adequação, a aceitabilidade e a qualidade técnico-científica do cuidado.

A avaliação de qualidade de serviços de saúde requer a pré-seleção de seus componentes e, por conseqüência, de seus valores de julgamento. A seleção dos componentes condiciona e determina o desenvolvimento de parâmetros e critérios. O produto da avaliação variará em função do número de dimensões e da precisão com a qual seu desempenho será explorado.

A acessibilidade às ações e serviços de saúde

Em um trabalho de revisão dos estudos de avaliação qualitativa dos serviços de saúde, Campos et al. (1990) apontam a existência de duas vertentes principais que se baseiam na acessibilidade como metodologia de avaliação da qualidade do cuidado. A primeira delas relaciona a eqüidade do acesso com as características da população (renda familiar, cobertura previdenciária, atitudes frente ao cuidado médico) ou do sistema de saúde (distribuição e organização dos serviços, relações de poder etc.). A segunda vertente relaciona a avaliação do acesso aos indicadores de resultado da passagem do indivíduo pelo sistema (padrões de utilização e de satisfação). Pesquisadores desta última vertente afirmam que esta metodologia permite "validações externas" da importância do sistema e das características individuais.

Segundo Donabedian (1973), existem dois aspectos da acessibilidade a serem distinguidos: o sócio-organizacional e o geográfico. Estão incluídos nos aspectos sócio-organizacionais os atributos referentes aos recursos e à organização, que podem facilitar ou dificultar os esforços do cliente em obter cuidado. Já a acessibilidade geográfica refere-se ao "isolamento espacial", uma função do tempo e da distância física a ser percorrida pelo paciente para a obtenção do cuidado.

Autores como Garro \& Young (1983), que utilizam a antropologia como referencial metodológico, enfatizam que a acessibilidade ao serviço de saúde é o fator primordial à sua utilização, superando mesmo barreiras étnicas e culturais.

Davis (1991) revisou os principais desenvolvimentos nas últimas duas décadas na literatura de investigação em serviços de saúde, sobre ineqüidade e acesso a serviços de atenção à saúde. Aponta um importante fator na sustentação de políticas de saúde nos Estados Unidos que visavam à expansão de programas de cuidado à saúde para a pobreza e outros grupos populacionais vulneráveis nos anos 60 e 70: o reconhecimento de que a situação de saúde e a utilização de serviços variam significativamente dependendo da renda pessoal, raça e localização geográfica.

As análises iniciais do impacto que programas como "Medicare", "Medicai d" e centros de saúde comunitários tiveram no incremento do acesso a serviços de saúde, para o pobre e o idoso, reafirmaram a importância desses programas. Destacam-se entre os tipos de estudo desse período: exames de conexão entre status sócio-econômico e mortalidade; inquéritos abordando diferenças sócio-econômicas na utilização de serviços de atenção à saúde; métodos econométricos para análise da utilização de serviços de atenção à saúde e investigações de impacto do programa sobre os beneficiá- 
rios. Davis (1991) também menciona estudos de utilização de serviços de saúde através de análise multivariada dos fatores que afetam o uso de cuidado ambulatorial e hospitalizações de curto prazo, baseados em entrevistas realizadas com pacientes. Outro tipo de estudo utilizado foi a investigação da relação entre situação de saúde e utilização de serviços, comparando beneficiários desses programas com outros pacientes.

Vários estudos examinaram especificamente barrei ras de acesso aos serviços de saúde para os não-segurados, mostrando que os grupos sem cobertura experimentaram maiores dificuldades na obtenção de serviços de saúde. Em um artigo de revisão, Davis et al. (1981) assinalavam que pobres, minorias e adultos jovens continuavam sendo os grupos com maior probabilidade de não serem cobertos por seguro. As pessoas com seguro de saúde receberam $54 \%$ mais cuidado ambulatorial e $90 \%$ mais cuidado hospitalar do que aqueles sem cobertura. Schlesinger et al. (1987) investigaram o impacto da competição e a pressão por contenção de custos no acesso ao cuidado. Os autores acreditam que a tendência de grandes pressões sobre os custos levaria ao decréscimo de acesso para os não-segurados. Hadley et al. (1991) encontraram que não-segurados que são hospitalizados têm de $44 \%$ a $124 \%$ maior risco de mortalidade hospitalar no período da admissão do que os cobertos por seguros privados. Os não-segurados tinham também menor probabilidade de receber procedimentos de alto custo ou alta sensibilidade.

A tendência em direção a estudos mais desagregados, que enfocam serviços específicos e a ligação entre o acesso a serviços de saúde e resultados na saúde, constitui-se no mais recente desenvolvimento da literatura nessa área temática (Davis, 1991). Um desses estudos (Lurie et al., 1984) encontrou uma acentuada deterioração no acesso a cuidados de saúde vivenciada por pessoas que estavam excluídas da cobertura de um programa de saúde. Verificou-se um aumento da incidência de diabetes e hipertensão não tratados. A probabilidade de morrer também foi significativamente maior para aqueles que perderam cobertura do que para o grupo-controle que manteve a cobertura.

Estudos de acessibilidade em indivíduos infectados pelo HIV

O aumento do número de pessoas infectadas pelo HIV, que evoluiram ou não para AIDS, demandando serviços de saúde é um desafio para a organização e distribuição desses serviços.
A dimensão que os serviços devem adquirir ainda não é clara e estratégias de gerenciamento e desenvolvimento de cuidados para esses pacientes devem ser estabelecidas.

Menzer et al. (1992) analisaram o trabal ho desenvolvido por organizações não-governamentais, com o objetivo de prover educação para redução do risco, serviços de atenção e aconselhamento para pessoas com infecção pelo HIV. Os autores concluem que o desenvolvimento de programas de prevenção para pessoas infectadas pelo HIV devem se dirigir não apenas para aspectos comportamentais da doença, mas devem também contemplar as necessidades dos clientes referentes a serviços médicos e de suporte. De acordo com estes autores, a chave do sucesso é a capacidade de prover serviços altamente individualizados, a capacidade de oferecer acesso a indivíduos recentemente diagnosticados e a capacidade para identificar barreiras sociais e culturais para a busca e permanência nos serviços.

Davidson et al. (1992), enfocando pacientes infectados pelo HIV e que utilizaram um sistema público de atenção à saúde, estudaram a relação existente entre, por um lado, condições de emprego e barreiras financeiras e, por outro, a utilização de cuidados de saúde e medidas de condições de saúde. Foram avaliadas as seguintes áreas: funcionamento físico, social e integral, fadiga, saúde mental e percepção geral da saúde. O modelo incluía estágio da doença, contagem de CD4, modo de transmissão, condições de emprego e barreiras financeiras ao cuidado. O estudo concluiu que, dos pacientes infectados pelo HIV, a utilização de cuidados de saúde é reduzida entre os que enfrentam barreiras financeiras para o cuidado, enquanto que aqueles que possuem emprego de tempo parcial apresentam medidas de condições de saúde significativamente mais altas. Esforços de intervenção precoce e melhores medidas de condições de saúde para pessoas infectadas pelo HIV podem ser obtidos através de incremento do acesso a cuidados de saúdee oportunidades ocupacionais.

Tillett \& Matsoukas (1992) investigaram a extensão, a natureza e as causas de conflitos associados com HIV na provisão de cuidados de saúde. Os autores concluíram que conflitos relacionados com medo e preconceito têm sido e continuarão sendo o principal problema na provisão de cuidados de saúde associados com HIV.

Hidalgo et al. (1990) examinaram diferenças nas tendências seculares a partir da introdução de tratamento com zidovudina (ZVD) e pentamidina; avaliaram os efeitos de risco de 
HIV, etnicidade e capacidade de pagar sobre o acesso a esses tratamentos e determinaram o impacto do acesso a estes medicamentos sobre a sobrevivência. Os resultados encontrados revelaram diferenças significativas na probabilidade de receber tratamento com os medicamentos estudados. Os autores concluem que os achados do estudo reforçam relatos anteriores de incremento da sobrevivência entre pessoas tratadas com ZVD ou pentamidina. Também concluem que existem evidências que sugerem disparidade no acesso a esses tratamentos e que a provisão eqüitativa de tratamentos para AIDS é necessária para garantir que incrementos na sobrevivência sejam conseguidos por todos os segmentos da população infectada com HIV.

Rosemberg et al. (1990) investigaram se os efeitos propostos por terapias profiláticas na incidência de AIDS variam por comportamento de risco, raça ou geografia. Os resultados encontrados são consistentes com a hipótese de que a terapia profilática tem reduzido a incidência de AIDS. Porém, o estudo demonstrou que o acesso à terapia é desigualmente distribuído e que a ampliação do acesso a tratamentos atualmente disponíveis, como o AZT, beneficiaria especialmente grupos com baixa cobertura de atenção, "minorias" e pessoas diagnosticadas em locais não situados nas principais áreas metropolitanas.

Solomon et al. (1992) examinaram as contribuições independentes do status sorológico para o HIV, sintomas clínicos, contagem de céIulas CD4 e seguro de saúde para a utilização de serviços de saúde entre usuários de drogas injetáveis. O estudo constatou que a mais importante variável preditiva de utilização de serviços era a presença de dois ou mais sintomas clínicos relacionados com HIV. O status sorológico positivo isoladamente ou conhecimento prévio de contagem baixa de células CD4 não estavam significativamente associados com o uso de serviços de saúde. De acordo com os autores, tais dados sugerem que os usuários de drogas injetáveis que são soropositivos para o HIV não estão recebendo os cuidados preventivos recomendados, sendo necessários esforços adicionais para garantir sua participação nos protocolos vigentes para tratamento precoce de portadores do HIV assintomáticos.

Stein et al. (1991) avaliaram fatores sócioeconômicos que determinam a disponibilidade de ZVD em pessoas sintomáticas infectadas pelo HIV. Os resultados do estudo mostraram que as pessoas classificadas nas categorias: sexo masculino, com seguro, cor branca, não-usuários de drogas injetáveis e com história de um episódio de pneumonia por P. carinii tinham maior probabilidade de terem o ZVD disponível do que, respectivamente, as pessoas classificadas nas categorias: sexo feminino, sem seguro, cor “não-branca”, usuários de drogas injetáveis e sem história de qualquer epsódio de pneumonia por P. carinii. Conclui-se que aqueles grupos que se encontram tradicionalmente em desvantagem têm menos acesso ao ZVD.

Fife \& Mode (1992) observaram que, desde 1987, o incremento anual da incidência de AIDS entre homens com práticas homossexuais e bissexuais tem diminuido, mas ocorre o inverso quando se enfocam outros comportamentos de risco. Afirmam que, apesar de evidências indiretas sugerirem que a mudança na incidência está relacionada com o cuidado médico para a infecção pelo HIV, adiando o aparecimento da AIDS, outras explicações são também possíveis. Para examinar essa mudança de incidência sob uma perspectiva diferente, aqueles autores classificaram os moradores de Filadélfia (EUA) com AIDS pelo seu rendimento per capita. Encontraram que a incidência de AIDS cresceu acentuadamente no terço de menor rendimento; mostrou crescimento contínuo, porém menor, depois de 1987 no terço médio; e manteve o nível depois de 1987 no terço de maior rendimento. Esta relação entre rendimento e mudanças de incidência persistiu depois da estratificação por raça ou modo de infecção pelo HIV. Verificaram que o rendimento estava associado com seguro médico privado no período de diagnóstico de AIDS (59\% possuíam seguro privado no terço superior e $24 \%$ no terço inferior) e com o tempo médio de sobrevivência depois do diagnóstico de AIDS (467 dias no terço superior e 359 dias no terço inferior). Os autores concluem que essas observações são consistentes com os benefícios gerados pelo tratamento médico que são obtidos pelo terço de maior rendimento mas não alcançados pelo terço de menor rendimento.

Alguns fatores associados com a satisfação do paciente entre pessoas sintomáticas infectadas pelo HIV foram estudados por Stein et al. (1993). Apontam a facilidade de acessar cuidados médicos como um dos mais importantes focos de satisfação do usuário. Os resultados desse estudo revelam que um número de variáveis tem um efeito significativo sobre a satisfação com o acesso ao cuidado médico. As condições de cobertura por seguro de saúde têm notáveis efeitos. Em contraste, características demográficas tiveram um efeito mínimo na satisfação com acesso. A intensidade dos sintomas foi negativamente relacionado, de forma significativa, com todos os resultados de satis- 
fação. O local usual do cuidado também influenciou dramaticamente a satisfação com o acesso. Também é digno de nota o fato de que pessoas com maior quantidade e/ ou freqüência de sintomas relacionados ao HIV tiveram menores scores de satisfação em todas as dimensões da satisfação mensuradas por meio das escalas utilizadas pelo estudo. A gravidade do sintoma foi fortemente relacionada com a freqüência de visitas ambulatoriais, a maior demanda de serviços e a maior proximidade com que os pacientes podem experienciar problemas relacionados com o sistema de cuidados à saúde, como, por exemplo, a demora. Os autores concluem que a conveniência do cuidado e outros aspectos do acesso são obviamente críticos na ajuda de pessoas mais sintomáticas.

Estudos recentes têm enfocado a acessibilidade a cuidados de saúde como um indicador apropriado para investigar violação de direitos humanos relacionados com HIV/AIDS. Para Osorio-Ramirez (1994), esse problema vinculase à incapacidade dos governos de reduzir o impacto que a pandemia tem provocado. O autor alerta para a influência da discriminação e do estigma na adoção de políticas e processos de tomada de decisão e enfatiza, entre outros exemplos, os poucos recursos disponíveis para enfrentar adequadamente os custos da pandemia no mundo. Panebianco et al . (1994), revisando as 1.543 reclamações referentes a violações de direitos humanos encaminhadas ao Conselho Nacional de AIDS, México D.F., nos anos de 1992 e 1993, conclui que as violações ocorrem mais freqüentemente na procura por atenção médica, e incluem recusa de atendimento, atenção inapropriada e veto à medicação. A discriminação contra pacientes com infecção pelo HIV expressa pela recusa de atendimento por parte dos trabalhadores em saúde étambém relatada em $79 \%$ dos casos de um estudo que entrevistou 212 pacientes provenientes de cada região da Itália (D'Andrea et al., 1994). Abordando essa mesma questão, Charbonneau et al. (1994) encontraram um percentual de $21 \%$ de recusa de tratamento odontológico entre 224 indivíduos infectados pelo HIV e residentes na região metropolitana de Montreal.

\section{Considerações finais}

O estudo da acessibilidade a cuidados e serviços de saúde, buscando caracterizar barreiras financeiras, organizacionais, ecológicas, sócioculturais e de condutas, foi, nas últimas quatro décadas, e continua a ser o principal foco de investigação em serviços de saúde. Entretanto, não é esta a realidade no espaço latino-americano e mais especificamente no Brasil, onde este problema é um dos mais discutidos, e menos analisados, dentro deste campo de investigação.

Não é de agora que se vem planteando políticas destinadas a aumentar esta acessibilidade, que se pressupõe insuficiente para amplas camadas da população. Sem necessidade de retrocedermos muito no tempo, desde os anos 70, a ampliação de cobertura (postulada na Reunião de Ministros de Saúde realizada em outubro de 1972 em Santiago do Chile) é um elemento sempre presente nas diversas políticas de saúde sub-regionais.

Apesar disto, muito pouco é o que objetivamente se tem avançado no conhecimento desta problemática. Assim, continuam sem resposta certas perguntas, tais como as seguintes: - Para uma mesma origem da demanda (ou seja, para algumas patologias e estágios destas bem definidos, como gravidade, visibilidade da afecção) existem diferenças pelas variáveis sexo, idade, lugar de resi dência, status sócio-econômico? Que tipo ou conjunto de variáveis está mais associado com a maior (menor) acessibilidade de um conjunto social bem definido? - Admitindo que a acessibilidade é diferencial por estratos de população, quer dizer, reconhecendo como verdadeiro o fato de que existe uma relação inversa entre necessidades não satisfeitas e acesso aos serviços de saúde (afirmação tautológica, mas não óbvia), essa desigualdade é da mesma magnitude frente às diversas demandas que integram o espectro da saúde (de consulta, de internações, de medicamentos etc.)?

- Que papel joga a complexidade dos serviços de saúde na acessibilidade? A acessibilidade é - como se pressupõe - inversamente proporcional à complexidade (quanto mais complexo um serviço, menor o acesso da população ao mesmo)?

- Pode-se supor, com uma base empírica aceitável, que existe uma acessibilidade diferencial segundo a dependência administrativa dos serviços, e que nesse acesso diferencial o fator econômico joga um papel decisivo (por exemplo, os serviços pagos são menos acessíveis que os serviços gratuitos, mantendo iguais todos os outros fatores)? 
- Quais elementos que cada um dos conjuntos sociais leva em consideração para efetuar suas demandas por serviços de saúde?

Poderíamos seguir listando perguntas, mas cremos que as já enunciadas são uma mostra suficiente do grau de desconhecimento existente sobre a maior parte dos temas relevantes para a administração dos serviços de saúde, assim como, também, para a definição de políticas sanitárias que não surjam apenas de postulados (explícitos ou não), e, sim, principalmente, do conhecimento e análise da realidade regional e local.

$\mathrm{Na}$ busca deste conhecimento, pode-se constatar a crescente importância de investigações que se ocupam das ineqüidades nos resultados de saúde e acesso a serviços, contribuindo ao debate sobre políticas públicas. Documentação de disparidades no uso de serviços, investigações sobre o impacto adverso de medidas de contenção de custos em programas de saúd de e investigações sobre as conseqüências da existência de barreiras para o cuidado nos resultados de saúde são alguns exemplos de estudos que têm subsidiado a defesa da meIhoria de sistemas de saúde.

Neste contexto, a epidemia causada pelo HIV, que pode levar à AIDS, adquire uma dimensão singular. Em primeiro lugar, pelo te- mor gerado pela variedade de características da doença, até o presente momento incurável e de mortalidade universal. Em segundo Iugar, pela inequívoca responsabilidade pública que a doença acarreta. E, em terceiro lugar, pela magnitude do problema e pelo impacto que a doença causa sobre o sistema de atenção à saúde.

O quadro assim delineado certamente exigirá do poder público crescentes esforços visando à prevenção e ao controle da doença. Para isto, é necessário um melhor conhecimento no que se refere tanto à situação da epidemia, quanto às suas conseqüências sobre o sistema de saúde.

A epidemia de AIDS/ HIV tem marcado fortemente a prática das instituições e dos profissionais de saúde, além de trazer para o conjunto da assistência, a existência de ineqüidades na distribuição das ações e serviços de saúde.

Assim, é inquestionável, no caso específico dessa epidemia, a necessidade de compreender as implicações das diversas barreiras de acesso aos serviços, considerando-se os custos sociais e humanos da infecção pelo HIV, as necessidades e demandas dos indivíduos infectados e as conseqüências que o não-atendimento em tempo hábil pode ter para estes indivíduos.

\section{Referências}

ADAY, L. A. \& ANDERSEN, R., 1974. A framework for the study of access to medical care. Health Services Research, 9:208-20.

BANCO MUNDIAL, 1993. Relatório sobre o Desenvolvimento Mundial. Investindo na Saúde. Washington D.C.: Banco Mundial.

BIANCARELLI, A.,1993. Pesquisa revela o medo da AIDS no país. Fol ha deSão Paulo, 11 de julho, Caderno Cotidiano.

CAMPOS, F. E.; ACURCIO, F. A.; REIS, E. F. J. B.; SANTOS, F. P.; LEITE, M. T. T.; LEITE, M. L. C.; CHERCHIGLIA, M. L. \& SANTOS, M. A., 1990. Avaliação da qualidade dos serviços de saúde: notas bibliográficas. Cadernos de SaúdePública, 6:50-61.

CASTILHO, E. A.; CHEQUER, P. \& STRUCHINER, C. J., 1992. AIDS no Brasil. Informe Epidemiológico do SUS/Fundação Nacional de Saúde, 3:115-124.

CHARBONNEAU, A.; MATHEUX, B. \& BÉLAND, F., 1994. Is there discrimination against people with HIV/AIDS when they seek dental care? X International Conference on AIDS, Abstracts. p.417. Yokohama.
CHORNY, A.; DURAN, L.; GOLDBAUM, M.; GONZÁLEZ, G. \& ROSSI, S., 1990. Investigacion y sistemas locales de salud. In: Los Sistemas Locales de Salud (J. M. Paganini \& R C. Mir, eds.), pp. 450455. Washington, D.C.:Ed. Organizacion Panamericana de Salud.

D'ANDREA, C.; VACCHER, E.; SPINA, M.; ERRANTE, D.; BERNARDI, D.; SIMONELLI, C.; NASTI, G. \& TIRELLI, U., 1994. Discrimination against patients with HIV infection by health care workers. $X$ International Conference on AIDS, Abstracts. p.417. Yokohama.

DAVIDSON, A.; RIETMEIJER, C.; HAGGLUND, B.; PAULSON, A.; ST. JOHN, M. \& COHN, D., 1992. Relation of social factors to health care utilization and health status assessment in HIV-infected patients. VIII Internatinal Conference on AIDS, Abstracts. p.510. Amsterdã.

DAVIS, K.; GOLD, M. \& MAKUC, D., 1981. Access to health care for the poor: does the gap remain? Annual Review of Public Health, 2:159-182. 
DAVIS, K., 1991. Inequality and access to health care. The Milbank Quartely, 69:253-273.

DE GEYNDT, W., 1970. Five approaches for assessing the quality of care. Hospital Administration, 1:5-42.

DONABEDIAN, A., 1966. Evaluating the quality of medical care. Milbank Memorial Fund Quartely, 44:166-206.

DONABEDIAN, A., 1973. Aspects of Medical CareAdministration. Cambridge: Haward University Press.

DONABEDIAN, A., 1986. La investigación sobre la calidad de la atención médica. Salud Publica de Mexico, 29:324-327.

DONABEDIAN, A., 1990a. Garantía y Monitoría de la Calidad dela Atención Médica. M éxico D.F.: Ed. Instituto Nacional de Salud Publica.

DONABEDIAN, A., 1990b. The seven pillars of quality. Archives of Pathology and Laboratory Medicine, 114:1115-1118.

FIFE, D. \& MODE, C., 1992. AIDS incidence and income. Journal of Acquired Immunodeficiency Syndrome, 5:1105-1110.

GARRO, L. Y. \&YOUNG, J. C., 1983. Atención de salud en minorias etnicas rurales. Algunas observaciones antropologicas. Boletin dela Oficina Sanitaria Panamericana, 95:333-344.

GUIM ARÃES, M. D. C. \& CASTILHO, E. A., 1993. Aspectos epidemiológicos da AIDS/HIV no Brasil. Revista da Soci edade Brasilei ra de Medicina Tropical, 26:101-111.

HADLEY, J.; STEINBERG, E. P. \& FEDER, J., 1991. Comparison of uninsured and privately insured hospital patients. Journal of the American Medical Association, 265:374-379.

HAYES, R., 1992. The Global Epidemiology of HIV and AIDS. Lecture Series on Public Health Issues 1992/ 3. Londres: London School of Hygiene and Tropical Medicine. (mimeo.)

HIDALGO, J.; SUGLAND, B.; MOORE, R. \& CHAISSON, R. E., 1990. Access, equity, and survival: Use of ZVD and Pentamidine by persons with AIDS. VI International Conference on AIDS, Abstracts, p.148, São Francisco.

LURIE, M.; WARD, N. B.; SHAPIRO, M. F. \& BROOK, R. $H .$, 1984. Termination from Medi-Cal: does it affect health? New England Journal of Medicine, 311:480-484.

MENZER, J. A.; GAMBRELL, A.; SCHINDLER, S.; VALDISSERR, R.; WEST, G. \& HINMAN, A. R., 1992. Providing prevention/ risk reduction to persons with early HIV disease by non-governmental, community based organizations (NGO/CBOs). VIII International Conference on AIDS, Abstracts. p. 435, Amsterdã.
MS (Ministério da Saúde), 1995. Programa nacional de doenças sexualmente transmissíveis/AIDS. AIDS Boletim Epidemi ológico, 1:6-9.

OSORIO-RAMIREZ, M.A., 1994. Human rights violations as a barrier to effective policies in prevention and care. $\mathrm{X}$ International Conference on AIDS, Abstracts. p.50. Yokohama.

PANEBIANCO, S.; DEL RIO, C.; BAÉZ-VILLASEÑOR, J.; URIBE, P. \& MORALES, G., 1994. Human rights violations and AIDS: Two parallel public health epidemics. X International Conference on AIDS, Abstracts. p.63. Yokohama.

ROSENBERG, P. S.; GAIL, M. H.; SCHRAGER, L.; GOEDERT, J. J. \& BIGGAR, R. J., 1990. Differential access to therapy may explain AIDS incidence trends in subgroups. VI International Conference on AIDS, Abstracts. p.291. São Francisco.

SCHLESINGER, M.; BENTKOVER, J.; BLUMENTAL, D.; MUSACCHIO, R. \& WILLER, J., 1987. The privatization of health care and physicians' perceptions of access to hospital services. The Milbank Quartely, 65:25-28.

SOLOMON, L.; FRANK, R.; VLAHOV, D. \& ASTEMBORSKI, J., 1992. Utilization of health services in a cohort of intravenous drug users with known HIV-1 serostatus. American Journal of Public Health, 81:1285-1290.

SOMERS, A. R., 1971. Health Care in Transition: Directions for the Future. Chicago: Hospital Research and Educational Trust.

STEIN, M. D.; PIETTE, J.; MOR, V.; WACHTEL, T. J .; FLEISHMAN, J.; MAYER, K. H. \& CARPENTER, C. C., 1991. Differences in access to Zidovudine (AZT) among symptomatic persons. Journal of General Internal Medicine, 6:35-40.

STEIN, M. D.; FLEISCHMAN, J.; MOR, V. \& DRESSER, M., 1993. Factors associated with patient satisfaction among symptomatic HIV-infected persons. Medical Care, 31:182-188.

TILLETT, G. \& MATSOUKAS, K., 1992. The resolution of HIV-related conflict in the provision of health care. VIII International Conference on AIDS, Abstracts. p.140. Amsterdã.

VUORI, H., 1988. Strategies for Improving the Quality of Health Care. Rio de Janeiro: Escola Nacional de Saúde Pública, Fundação Oswaldo Cruz. (mimeo.) 